\title{
Modeling AGN outbursts from supermassive black hole binaries
}

\author{
T. Tanaka ${ }^{\mathrm{a}}$ \\ Max-Planck-Institute for Astrophysics, Karl-Schwarzschild-Strasse 1, 85741 Garching bei Muenchen, Germany
}

\begin{abstract}
When galaxies merge to assemble more massive galaxies, their nuclear supermassive black holes (SMBHs) should form bound binaries. As these interact with their stellar and gaseous environments, they will become increasingly compact, culminating in inspiral and coalescence through the emission of gravitational radiation. Because galaxy mergers and interactions are also thought to fuel star formation and nuclear black hole activity, it is plausible that such binaries would lie in gas-rich environments and power active galactic nuclei (AGN). The primary difference is that these binaries have gravitational potentials that vary - through their orbital motion as well as their orbital evolution - on humanly tractable timescales, and are thus excellent candidates to give rise to coherent AGN variability in the form of outbursts and recurrent transients. Although such electromagnetic signatures would be ideally observed concomitantly with the binary's gravitational-wave signatures, they are also likely to be discovered serendipitously in wide-field, high-cadence surveys; some may even be confused for stellar tidal disruption events. I discuss several types of possible "smoking gun" AGN signatures caused by the peculiar geometry predicted for accretion disks around SMBH binaries.
\end{abstract}

\section{INTRODUCTION}

Given that galaxies assemble hierarchically and that nearly every massive galaxy appears to host a supermassive black hole $(\mathrm{SMBH})$ in its nucleus $[1,2]$, it is inevitable that they go through stages in which they harbor multiple SMBHs. Dissipation of gravitational energy via dynamical friction should form bound SMBH binaries, which become increasingly compact as they interact with ambient stars and gas before inspiraling and coalescing by emitting gravitational radiation [3]. Such binaries should be common throughout cosmic time [4-8] and are expected to be strong sources of gravitational radiation during inspiral and merger. Because galaxy mergers are also thought to trigger active galactic nuclei (AGN) and quasars [9-11], it is plausible that $\mathrm{SMBH}$ binaries tend to reside in gas-rich environments and power nuclear activity.

The possibility that SMBH binaries can emit unique, "smoking gun" electromagnetic signatures has attracted attention because of the immense scientific potential of observing such signatures concomitantly with the gravitational wave signatures [12-16]. However, conspicuous, coherent emission from SMBH binaries could also be detected by electromagnetic searches alone (as with the candidate binary OJ 287 [17]), either by targeted monitoring of AGN $[18,19]$ or in wide-field, high-cadence surveys [20,21].

A particularly promising mechanism for producing such signatures is the geometry of a coplanar, circumbinary accretion disk, namely the presence of an annular gap or a central quasicircular cavity. ${ }^{1}$ Here, I discuss three types of observable features that may be produced by a central disk cavity: a softer thermal spectrum featuring intrinsically dim UV and X-ray emission (§2);

\footnotetext{
a e-mail: taka@mpa-garching.mpg.de

${ }^{1}$ For an overview of proposed electromagnetic signatures and their triggering mechanisms and timescales, see [22].
}

the evolution of this disk spectrum during and after the merger of the binary ( $\$ 3)$; and transient, quasiperiodic flares fueled by inward leakage of circumbinary gas into the cavity and onto one of the SMBHs ( $\$ 4)$.

\section{CIRCUMBINARY CAVITY}

The binary's tidal torques inject angular momentum into the surrounding accretion disk, so that the infall of the outer material is inhibited, creating an annular gap at a radius approximately twice the semimajor axis [23]. ${ }^{2}$ Because the gas inside the annulus is accreted much more rapidly than the binary evolves toward merger, the gap should evolve into a central, quasicircular cavity [25]; enough gas may leak into the cavity to form small accretion disks around one or both SMBHs [26].

In either case, because the innermost regions of accretion disks are where the highest-energy photons and the vast majority of the emergent flux is generated (independently of the disk viscosity mechanism), the presence of a central cavity leads to the disk having a softer spectrum and less bolometric output [27,28].

The presence of a cavity has two additional implications for the accretion rate of the circumbinary disk. First, the weakened emission mentioned above means that the accretion rate is not Eddington-limited in the usual sense. The outer accretion rate can be many times the fiducial "critical" value for a disk around a single SMBH, without producing sufficient radiation pressure to drive significant mass loss. Second, the inward mass flux at large radii is generally higher than the mass leakage rate into the cavity (see $\$ 4$ below). As such, the disk is not steady-state, with mass "piling up" just outside the cavity $[29,30]$.

\footnotetext{
${ }^{2}$ See [24] for detailed considerations of the conditions for gap formation (and non-formation).
} 


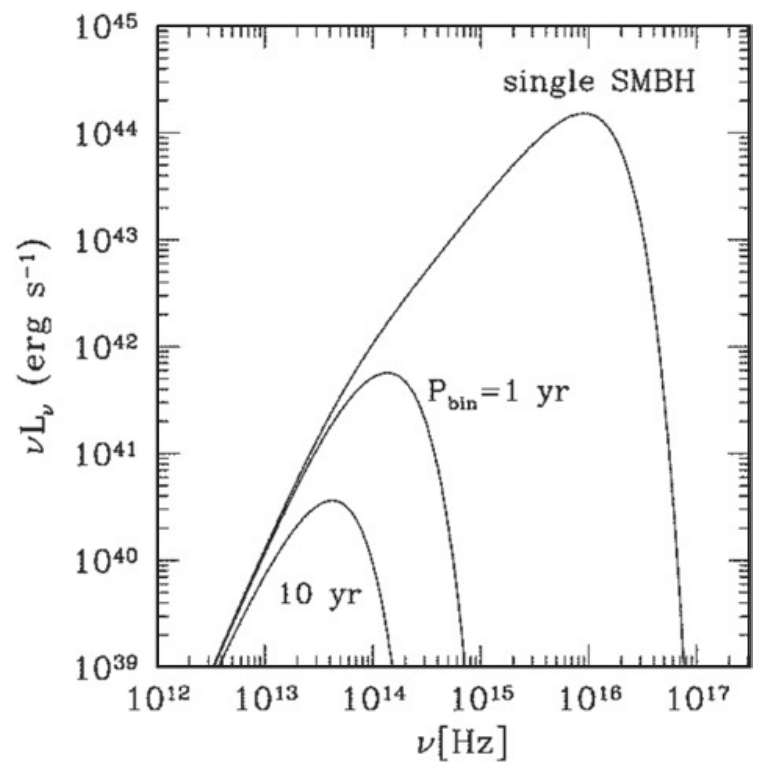

Figure 1. Thermal blackbody disk spectra, from top to bottom: a standard thin disk [32] around a single SMBH of mass $10^{7} \mathrm{M}_{\odot}$ and an accretion corresponding to $30 \%$ of the Eddington limit and a radiative efficiency $\epsilon=0.1$; the same disk around a binary with mass $10^{7} \mathrm{M}_{\odot}$, with the density profile truncated at twice the semimajor axis corresponding to of a binary orbital period $P_{\text {bin }}=1 \mathrm{yr}$; same, but with $P_{\text {bin }}=10 \mathrm{yr}$. The disk is assumed to be marginally gravitationally stable at large radii [33].

If the body emits as a blackbody, the color temperature $T_{\mathrm{ph}}$ at a given radius is given by

$$
\sigma_{\mathrm{SB}} T_{\mathrm{ph}}^{4} \approx \frac{9}{8} \dot{M} \Omega^{2},
$$

where $\sigma_{\mathrm{SB}}$ is the Stefan-Boltzmann constant, $\dot{M}$ is the local mass flux and $\Omega$ is the angular velocity of the gas (approximately Keplerian; see, e.g. [31]). Then, a circumbinary disk around a binary with period $P$ will be intrinsically dim at frequencies above

$$
v_{\mathrm{dim}} \sim 10^{14}\left(\frac{M}{10^{7} \mathrm{M}_{\odot}}\right)^{-1 / 4}\left(\frac{P}{\mathrm{yr}}\right)^{-1 / 2} \mathrm{~Hz},
$$

for a surface density corresponding roughly to $30 \%$ of the critical accretion rate onto a single SMBH. Figure 1 shows examples of circumbinary disk spectra truncated at twice the semimajor axis, as compared with a standard thin disk with a single central SMBH of the same mass and surface density normalization. The aforementioned mass "pile-up" (whose effects are minor) is neglected in the figure, as is the possible presence of a small accretion disk around either $\mathrm{SMBH}$; the latter would show up as a bump on the highfrequency end of the thermal spectrum [27].

\section{AFTERGLOW OF BINARY MERGER}

For most of the lifetime of the disk-binary system, the binary evolves sufficiently slowly so that the disk coevolves with it in a quasi-steady manner, maintaining the radial size of the cavity at $\sim 2 a[29,30,34]$. However,

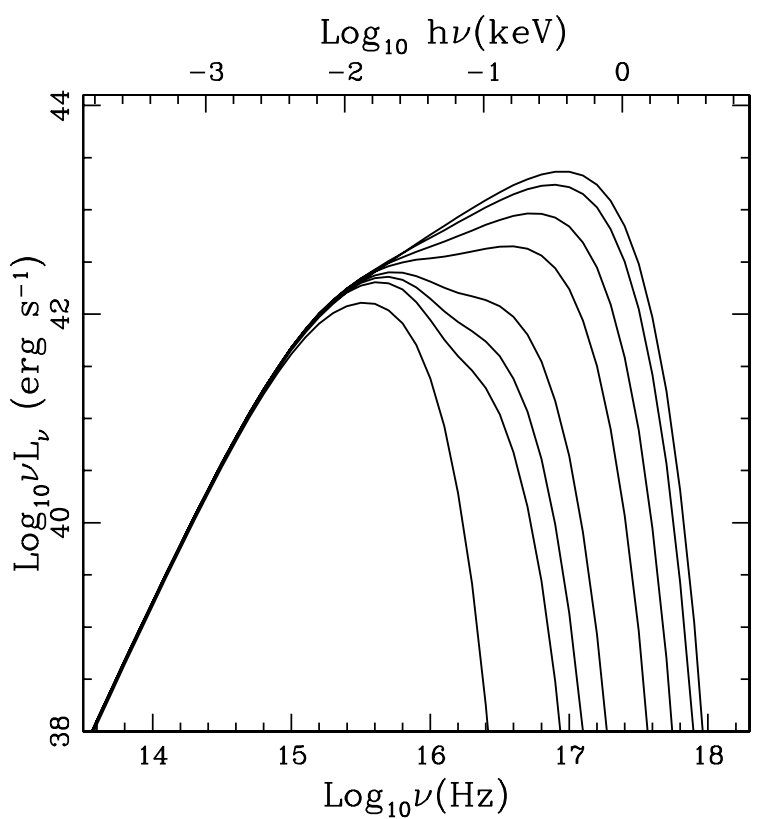

Figure 2. The spectral evolution of the circumbinary disk as it spreads viscously before and after the merger of an equal-mass binary with total mass $10^{6} \mathrm{M}_{\odot}$. From left to right: to 3 years before the merger; and 1 month, 1 year, 2 years, 5 years, 9 years, 20 years and 120 years after the merger. Adapted from [36].

at binary semimajor axis values of $a \sim 100 G M / c^{2}$, the binary begins to inspiral through gravitational-wave emission faster than the circumbinary disk can viscously spread $[25,35,36]$. The disk is left "frozen" as the binary merges inside the cavity, emitting a burst of gravitational waves. The disk - no longer inhibited by the binary's tidal torques - fills the cavity after a delay, evolving from a dim, spectrally soft source to a luminous disk whose thermal emission is dominated by UV and soft X-ray frequencies $[25,36,37]$. For SMBH binaries in the mass range $M \sim 10^{6} \mathrm{M}_{\odot}$, the afterglow evolves on timescales of years (Fig. 2).

The local mass flux of the gas,

$$
\dot{M}(R, t)=3 \pi v \Sigma\left(1+\frac{\mathrm{d} \ln v \Sigma}{\mathrm{d} \ln R}\right),
$$

can become very high as it fills the cavity, because of steep density and temperature gradients that were previously maintained by the binary torques; note that the gradient term inside the parentheses is zero in steady-state solutions. The mass fluxes are likely to be well above the critical rate, especially given the aforementioned point (\$2) that the diminished luminosity of the inner disk allows for very high surface densities. That is, the innermost disk may satisfy

$$
\dot{M}>3 \pi v \Sigma \gg L_{\text {Edd }} / c^{2}
$$

as it fills the cavity, with strong advective energy fluxes as in the slim-disk class of solutions of luminous accretion flows [38]. The gas may launch powerful outflows or a jet as it reaches the central SMBH [36].

Observing the source with gravitational as well as electromagnetic radiation would maximize the scientific yield. The position of the merging binary on the sky can 
be localized with a gravitational-wave detector such as LISA, and the spectral evolution of the AGN emission can be detected by follow-up monitoring. The monotonic brightening and spectral hardening of the source is likely to stand out, since (i) AGN typically exhibit little correlation between flux and spectral hardness in their variability [39], and (ii) X-ray-dim AGN are rare (perhaps less than $2 \%$ of the optically bright population $[40,41])$. Therefore, these afterglows could be discovered serendipitously by wide-field X-ray surveys that revisit the same patch of the sky, such as eROSITA - and, if some of the energy is reprocessed into the optical or near-infrared, then by LSST [21].

\section{TRANSIENT OUTBURSTS}

The last point is an interesting one, because the fraction of galaxies hosting nuclear SMBH binaries with subparsec separations is poorly constrained by observations. In principle, the fraction can be quite large, because the typical timescales it takes for binaries to reach the final, gravitational wave-emitting stage of evolution may be very long ("the final parsec problem"; [42]). SMBH binaries should most commonly have separations where their evolution is slowest; theoretical considerations suggest $\sim 10^{-3}-10^{-1} \mathrm{pc}[3,6]$, corresponding to orbital periods of years to centuries.

At these separations, the circumbinary disk (whose presence may be a prerequisite for some binaries to overcome the "final parsec" barrier), perhaps in a marginally gravitational stable state [33], would have a large cavity that prevents thermal emission above optical frequencies (eq. 2). That is, even if a circumbinary disk is present, it may not easily be detectable as an AGN.

Gas from the disk leaks into the cavity quasiperiodically in the form of discrete streams on nearly radial orbits, modulated by the binary's tidal torques [43]. The mass contained in an individual stream should be of order

$$
M_{\text {stream }} \sim f_{\text {leak }} \dot{M} P \sim 2.2 \frac{f_{\text {leak }}}{0.1} \frac{P}{100 \mathrm{yr}} \dot{m} \frac{M}{10^{7} \mathrm{M}_{\odot}} \mathrm{M}_{\odot},
$$

where $f_{\text {leak }}$ - found to be of order $1-10 \%$ in numerical simulations $[31,44]$ - is the fraction of the mass supply rate in the outer disk that enters the cavity and $\dot{m}$ is the ratio of the mass surface density just outside the cavity to the critical value in a steady-state disk around a single SMBH.

After the stream passes through pericenter near one of the SMBHs, it will self-intersect and shock. The bound material will circularize into a hot, optically thick annulus and viscously spread. The gas will begin to accrete in a slim-disk configuration (cf. [45]) before it can cool radiatively, producing transient $\mathrm{AGN}$-like emission in optical, UV and X-rays.

Figure 3 shows the bolometric, $u$-band $(365 \mathrm{~nm})$ and FUV (1500 ^) light curves of such an accretion event. In this particular calculation, the binary has a total mass of $10^{7} \mathrm{M}_{\odot}$ and an orbital period of $30 \mathrm{yr}$. The circularized accretion flow is modeled as an annulus of mass $1 \mathrm{M}_{\odot}$ initially at a radius $0.05 a$ from the secondary $\mathrm{SMBH}$

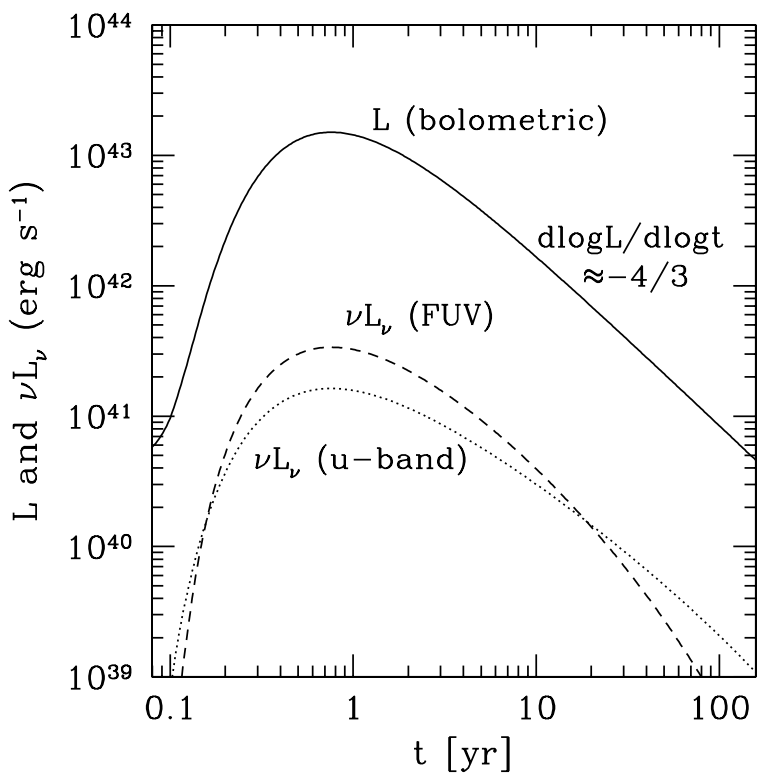

Figure 3. The bolometric (solid), $u$-band (365 nm; dotted) and

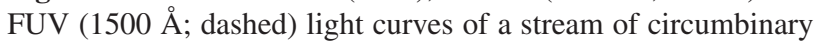
gas that circularizes and shock-heats before being accreted by the secondary SMBH $\left(2 \times 10^{6} \mathrm{M}_{\odot}\right)$. The bolometric light curve decays at late times with a power-law index $-4 / 3$. The binary in this model would produce a flare like this one every $\approx 30 \mathrm{yr}$.

with mass $2 \times 10^{6} \mathrm{M}_{\odot}$, and evolved using the exact diskevolution solutions derived in [46]. The annulus is shockheated so that its thermal energy is comparable to the Keplerian kinetic energy (satisfied if the shocks convert the gas orbits from nearly radial ones to nearly circular ones). The bolometric luminosity decays at late times as $\mathrm{d} \log L / \mathrm{d} \log t=-4 / 3$, but the decay rates in individual photometric bands deviate from this power law.

If the above scenario is realized in nature, then accreting SMBH binaries can produce optical, UV and Xray flares in otherwise quiescent galactic nuclei at cadences of years to centuries. Such flares may be mistaken for tidal disruptions of stars, given that the two phenomena are powered by the same underlying astrophysical process - i.e., the transient accretion of $\sim 1 \mathrm{M}_{\odot}$ of matter by a $\mathrm{SMBH}$ - and share similarities in emission energies and decay. However, a significant distinction is that the transient outbursts from leaked circumbinary gas would not be limited by SMBH mass in the way tidal disruption events are [47].

Because the event rate per galaxy is several orders of magnitude higher than the expected rate of tidal disruption events in galaxies without binary SMBHs $\left(10^{-5}-10^{-4}\right.$ per year per galaxy; e.g. [48]), if even a fraction of one percent of galaxies host accreting, hard SMBH binaries, the type of flares discussed here could be detected in significant numbers in surveys searching for tidal disruption events.

\section{CONCLUSIONS}

A central SMBH binary engine is capable of eliciting coherent AGN signatures in ways that a single SMBH engine cannot. The alteration of the central accretion 
disk by the binary's gravitational torques should make the thermal disk emission stand out, even in the absence of observed variability. In addition, the binary's orbital period and the gravitational-wave orbital evolution set two timescales that are observationally tractable. The viscous spreading of the circumbinary disk following a binary may appear to be an X-ray AGN turning on, i.e. as the "birth of a quasar." Before the merger, the orbital modulation of the gravitational torques presents a natural mechanism for feeding the central binary with discrete blobs of gas to produce luminous flares. Both types of proposed accretion events are expected to be optically thick and have large mass accretion rates and luminosities. Advective effects are likely to be important, and the luminous flares may be accompanied by powerful outflows or jets.

Because these outbursts produced by accretion flows that are coherent but intrinsically not steady-state, they could be used as laboratories of accretion physics, especially if the properties of the central SMBH engine (masses, spins, orbital parameters) can be determined with gravitational-wave observations or other methods. If the basic underlying theoretical predictions are correct, these luminous episodes should be observed in upcoming widefield, high-cadence surveys such as eROSITA and LSST.

I am grateful to the organizers for putting together an insightful and thought-provoking workshop. Much of $\S 4$ was conceived in the day before my contributed talk; an expanded discussion is being prepared for publication as a full journal article (Tanaka, in prep.). I am indebted to many people, particularly Linda Strubbe and Zoltán Haiman, for encouraging discussions. I thank my collaborators Kristen Menou and Zoltán Haiman; our work together is principally featured in $\S 3$.

\section{References}

[1] J. Kormendy, D. Richstone, ARAA, 33, 581 (1995)

[2] J. Magorrian, S. Tremaine, D. Richstone, R. Bender, G. Bower, A. Dressler, S. M. Faber, K. Gebhardt, R. Green, C. Grillmair. et al., AJ, 115, 2285 (1998), arXiv:astro-ph/9708072

[3] M. C. Begelman, R. D. Blandford, M. J. Rees, Nature, 287, 307 (1980)

[4] M. G. Haehnelt, MNRAS, 269, 199 (1994), astro$\mathrm{ph} / 9405032$

[5] K. Menou, Z. Haiman, V. K. Narayanan, ApJ, 558, 535 (2001), astro-ph/0101196

[6] Q. Yu, MNRAS, 331, 935 (2002), arXiv:astro$\mathrm{ph} / 0109530$

[7] M. Volonteri, F. Haardt, P. Madau, ApJ, 582, 559 (2003), astro-ph/0207276

[8] T. Tanaka, Z. Haiman, ApJ, 696, 1798 (2009), 0807.4702

[9] D. B. Sanders, B. T. Soifer, J. H. Elias, B. F. Madore, K. Matthews, G. Neugebauer, N. Z. Scoville, ApJ, 325, 74 (1988)

[10] L. Hernquist, Nature, 340, 687 (1989)

[11] R. G. Carlberg, ApJ, 350, 505 (1990)

[12] C. Cutler, PRD, 57, 7089 (1998), arXiv:grqc/9703068
[13] D. E. Holz, S. A. Hughes, ApJ, 629, 15 (2005), astro$\mathrm{ph} / 0504616$

[14] B. Kocsis, Z. Frei, Z. Haiman, K. Menou, ApJ, 637, 27 (2006), astro-ph/0505394

[15] E. S. Phinney, Finding and Using Electromagnetic Counterparts of Gravitational Wave Sources, in Decadal Survey White Paper (2009), Vol. 2010, p. No. 235

[16] J. S. Bloom, D. E. Holz, S. A. Hughes, K. Menou, Coordinated Science in the Gravitational and Electromagnetic Skies, in Decadal Survey White Paper (2009), Vol. 2010, p. No. 20

[17] H. J. Lehto, M. J. Valtonen, ApJ, 460, 207 (1996)

[18] M. Eracleous, J. P. Halpern, A. M. Gilbert, J. A. Newman, A. V. Filippenko, ApJ, 490, 216 (1997), arXiv:astro-ph/9706222

[19] M. Eracleous, J. P. Halpern, ApJ, 599, 886 (2003), arXiv:astro-ph/0309149

[20] Z. Haiman, B. Kocsis, K. Menou, Z. Lippai, Z. Frei, Class. Quantum Gravity, 26, 094032 (2009), 0811.1920

[21] T. Tanaka, Z. Haiman, K. Menou, AJ, 140, 642 (2010), 1004.5411

[22] J. D. Schnittman, Class. Quantum Gravity, 28, 094021 (2011), 1010.3250

[23] P. Artymowicz, S. H. Lubow, ApJ, 421, 651 (1994)

[24] B. Kocsis, Z. Haiman, A. Loeb, ArXiv e-prints (2012), 1205.5268

[25] M. Milosavljević, E. S. Phinney, ApJL, 622, L93 (2005), astro-ph/0410343

[26] K. Hayasaki, S. Mineshige, L. C. Ho, ApJ, 682, 1134 (2008), 0708.2555

[27] T. Tanaka, K. Menou, Z. Haiman, MNRAS, 420, 705 (2012), 1107.2937

[28] K. Gultekin, J. M. Miller, arXiv e-prints (2012), 1207.0296

[29] D. Syer, C. J. Clarke, MNRAS, 277, 758 (1995), arXiv:astro-ph/9505021

[30] P. B. Ivanov, J. C. B. Papaloizou, A. G. Polnarev, MNRAS, 307, 79 (1999), astro-ph/9812198

[31] A. I. MacFadyen, M. Milosavljević, ApJ, 672, 83 (2008), astro-ph/0607467

[32] N. I. Shakura, R. A. Sunyaev, A\&A, 24, 337 (1973)

[33] E. Sirko, J. Goodman, MNRAS, 341, 501 (2003), arXiv:astro-ph/0209469

[34] Y. T. Liu, S. L. Shapiro, PRD, 82, 123011 (2010), 1011.0002

[35] P. J. Armitage, P. Natarajan, ApJL, 567, L9 (2002), astro-ph/0201318

[36] T. Tanaka, K. Menou, ApJ, 714, 404 (2010), 0912.2054

[37] S. L. Shapiro, PRD, 81, 024019 (2010), 0912.2345

[38] M. A. Abramowicz, B. Czerny, J. P. Lasota, Szuszkiewicz E., ApJ, 332, 646 (1988)

[39] D. Grupe, H. Thomas, K. Beuermann, A\&A, 367, 470 (2001), arXiv:astro-ph/0012100

[40] R. R. Gibson, W. N. Brandt, D. P. Schneider, ApJ, 685, 773 (2008), 0808.2603

[41] O. Shemmer, W. N. Brandt, S. F. Anderson, A. M. Diamond-Stanic, X. Fan, G. T. Richards, D. P. Schneider, M. A. Strauss, ApJ, 696, 580 (2009), 0902.1366 
[42] D. Merritt, M. Milosavljević, Liv. Rev. Rel. 8, 8 (2005), arXiv:astro-ph/0410364

[43] P. Artymowicz, S. H. Lubow, ApJL, 467, L77+ (1996)

[44] Y. Ochi, K. Sugimoto, T. Hanawa, ApJ, 623, 922 (2005)
[45] L. E. Strubbe, E. Quataert, MNRAS, pp. 1532-+ (2009), 0905.3735

[46] T. Tanaka, MNRAS, 410, 1007 (2011), 1007.4474

[47] M. J. Rees, Nature, 333, 523 (1988)

[48] T. Alexander, Physics Reports, 419, 65 (2005), arXiv:astro-ph/0508106 